\title{
Predicción del rendimiento en cortes, hueso y grasa en búfalos de agua en Venezuela
}

\author{
Oscar Atencio-Valladares ${ }^{(1)}$, Nelson Huerta-Leidenz ${ }^{(1)}$, Argenis Rodas-González ${ }^{(2)}$ y Nancy Jerez-Timaure ${ }^{(1)}$
}

(1)Universidad del Zulia, Núcleo Agropecuario, Facultad de Agronomía, Dep. de Zootecnia, Apartado 15205, Maracaibo 4005, Venezuela. E-mail: ogatencio@hotmail.com, carnesluz@gmail.com, jerez.nancy@gmail.com (2)Universidad del Zulia, Núcleo Agropecuario, Facultad de Ciencias Veterinarias, Dep. de Producción e Industria Animal, Apartado 15252, Maracaibo 4005, Venezuela. E-mail: argenisrodas@yahoo.es

\begin{abstract}
Resumen - Los objetivos de este estudio fueron determinar la asociación entre rasgos de la canal y el rendimiento en cortes (RCD), rendimiento en hueso y recortes de grasa en búfalos de agua, y desarrollar ecuaciones predictivas para cada variable mencionada. Se utilizaron 48 búfalos de agua (24 castrados y 24 enteros), sacrificados a los 17, 19 y 24 meses de edad con mestizaje de razas Murrah y Mediterránea. Se evaluaron las variables de la canal y del rendimiento en cortes. Se realizaron: pruebas descriptivas, análisis de correlación, residuos y de regresión lineal múltiple. En los castrados, el acabado de grasa y la circunferencia del muslo explicaron la mayor variación en RCD. El espesor de grasa y el porcentaje de grasa renal fueron las variables mayormente asociadas con el rendimiento en hueso. En los enteros, el acabado de grasa y la longitud de la canal explicaron la mayor variación en RCD. El recorte de grasa se asoció más con acabado de grasa, y el rendimiento en hueso se asoció con la conformación. Las ecuaciones obtenidas lograron explicar más del 50\% de la variación del RCD. Las ecuaciones para recorte de grasa y rendimiento en hueso tuvieron mayor fuerza predictiva.
\end{abstract}

Términos para indexación: Bubalus bubalis, regresión, ecuaciones de predicción, rendimiento cárnico.

\section{Yield prediction of boneless cuts, bone and fat trimmings from water buffaloes in Venezuela}

\begin{abstract}
The objectives of this study were to determine the association between carcass traits and the percentage yield in boneless cuts (PDC), bone, and fat trimmings of water buffalves; and to develop predicted equations for these variables. Forty-eight crossbred Murrah-Mediterránea buffaloes (24 steers and 24 bulls) were used slaughtered at 17,19 and 24 months. Carcass and retail-yield-cut traits were evaluated. The following analyses were made: descriptive test, correlation, residual and multiple lineal regression. In castrated animals, subcutaneous fat and circumference round explained the majority of the variation in PDC. The dorsal fat thickness and the renal fat were the variables mostly associated with bone yield. In bulls, the subcutaneous fat and the carcass length explained most of the variation of PDC. The fat trimmings showed the highest correlation coefficient with the subcutaneous fat, and the bone yield was most correlated with the conformation. The equations obtained explained more than $50 \%$ of the variation in PCD, however equations for bone yield and fat trimmings showed a higher $\mathrm{R}^{2}$.
\end{abstract}

Term index: Bubalus bubalis, regression, retail yield cuts, predictive equations.

\section{Introducción}

La clasificación venezolana de reses está basada en el Venezuela (1997), el cual establece una clasificación primaria por clases o condiciones sexuales de ganado y una categorización de canales por su calidad o por índices de rendimiento, de carácter opcional, dentro de cada clase de ganado. En Huerta-Leidenz (2002), se describen las experiencias ganadas en investigaciones sobre el sistema de clasificación de canales bovinas en Venezuela. Es conocido que la aplicación del índice de rendimiento de este sistema (Venezuela, 1997) carece de basamento científico, ya que no se desarrolló con técnicas de regresión, y los trabajos de validación no han arrojado resultados promisorios (Malaver et al., 2000; Rodas-González, 2005). Tampoco se espera que el índice de rendimiento oficial funcione igual en cualquier clase de machos, pues aún con ecuaciones predictivas del rendimiento en cortes deshuesados (RCD), las relaciones entre variables (grado de asociación y signo de la correlación) son diferentes para castrados y enteros, debido principalmente a la relación músculo:grasa 
(Atencio, 2001). Lo anterior hace pensar que, a la fecha, no se cuenta en el país con un sistema confiable de clasificación de canales por rendimiento.

Las experiencias en el desarrollo de ecuaciones predictivas del rendimiento en cortes, basadas en técnicas de regresión múltiple, para clasificar por rendimiento carnicero, se han reportado en Estados Unidos (Abraham et al., 1980; Shackelford et al., 1995), Australia (Hopkins \& Roberts, 1995), Europa (Muldowney et al., 2001), Asia (Lee et al., 2005), Latinoamérica (Jardim et al., 1991; Amador-Gómez \& Palacios-Gómez, 1994; Atencio, 2001; Schindler et al., 2004). Sin embargo, estas experiencias también han servido para demostrar que las ecuaciones obtenidas para un determinado grupo comercial de ganado vacuno, muchas veces no son extrapolables a otras regiones con animales en diferentes condiciones tanto intrínsecas como ambientales.

Las canales bufalinas son introducidas al circuito cárnico sin hacer distinción de la especie que se comercializa y por ende, dichas canales son clasificadas bajo el mismo sistema imperante para el ganado vacuno, a pesar de ser especies biológicamente diferentes. Para búfalos, no se puede pretender utilizar ecuaciones desarrolladas para ganado vacuno, que a pesar de ser comercializado como bovino, presenta diferencias sustanciales en rendimiento con el ganado vacuno.

El presente estudio utilizó el primer banco de datos con búfalos castrados y enteros en Venezuela, para determinar el grado de asociación entre rasgos de la canal y RCD, rendimiento porcentual en hueso (PHUESO) y recortes de grasa (PGRASA), y desarrollar ecuaciones de predicción para cada una de las variables dependientes mencionadas dentro de cada condición sexual.

\section{Material y Métodos}

Se utilizaran 48 búfalos de agua (24 machos castrados y 24 enteros) mestizos de las razas Murrah y Mediterránea, producidos en un hato de los llanos centrooccidentales venezolanos, sacrificados en forma seriada a los 17,19 y 24 meses de edad.

La primera fase de campo (hasta el destete a los 7 meses aproximadamente) comenzó en el hato "Los Cocos", ubicado en la ribera norte del Río Arauca, $60 \mathrm{~km}$ al oeste de la población El Yagual, Distrito Achaguas, Estado. Apure. La pluviosidad en este hato de cría es de $1.150 \mathrm{~mm}$, con un ciclo de lluvia de Mayo a Noviembre. El rebaño de búfalos de agua (búfalas y bucerros) estaba ubicado en la zona más baja del hato (sabanas naturales inundables, arcillosas, de fertilidad moderada y $\mathrm{pH}$ ácido), donde predominaban Paspalum fasciculatum, Himenachne amplexicanlis y Leersia hexandra. Las crías permanecieron con sus madres todo el tiempo hasta el destete y recibieron vacunas y antiparasitarios simultáneamente. La mitad de los machos destetados fueron castrados y luego todos los animales fueron enviados a otro hato ("Charcote"), ubicado en paralelo a la carretera Las Vegas-Tirado, $12 \mathrm{~km}$ al sur de San Carlos, Estado Cojedes, para completar la fase de levante y ceba y cumplir con las matanzas predeterminadas a los 17 meses (en promedio 517 días), 19 meses (en promedio 602 días), y 24 meses de edad (en promedio 736 días), aproximadamente. La zona de levante y ceba corresponde a sabanas altas, de suelo franco arcilloso, de fertilidad moderada, ligeramente ácidos. Los pastos predominantes eran Brachiaria humidicola, Brachiaria decumbens y Paspalum plicatulum. Al completar las edades asignadas de matanza, los animales preseleccionados al azar eran pesados en horas de la mañana, embarcados para su traslado al matadero de Valencia, Edo. Carabobo, con un periodo de ayudo que osciló entre 12-18 horas. En cada edad de matanza se sacrificaron 16 búfalos ( 8 enteros y 8 castrados), para un total de 48 animales en el ensayo.

Los animales se sacrificaron en una planta beneficiadora comercial conforme a las regulaciones establecidas. El desuello se efectuó en forma manual, y la grasa pélvica, la perirrenal y la cardiaca (grasa interna) no fueron retiradas. El peso de la canal caliente se tomó al final de la línea de faena, en la estación de clasificación, luego de la división de la canal y el lavado de las medias canales. A fin de precisar el peso de la canal caliente, no se descontaron los $5 \mathrm{~kg}$ por res, supuestamente por mermas en refrigeración, que estipula el procedimiento de clasificación del Ministerio de Agricultura y Tierras. A las 24 horas de frío post mortem, las canales fueron evaluadas según el Venezuela (1997), para distintas características cualitativas y de rendimiento.

Después de 48 horas de almacenamiento en cámaras de refrigeración, las canales se redujeron a cortes de carnicería para venta al detal, de acuerdo al sistema de despiece venezolano, atendiendo a las normas de la Comisión Venezolana de Normas Industriales (1982); se removió la grasa subcutánea en exceso, cuando la hubo, dejando un máximo de espesor de grasa de $6,4 \mathrm{~mm}$ en el corte, con las siguientes modificaciones: los cortes 
cogote y solomo abierto no fueron separados, es decir, el peso del solomo abierto registrado incluyó también el cogote. Asimismo, los cortes solomo de cuerito grueso (porción costal) y solomo de cuerito delgado (porción lumbar) no fueron separados, por lo que se tomó el peso de la pieza completa del solomo, registrada como "solomo de cuerito".

El corte del pecho se realizó a sierra, en la porción proximal de las costillas, cerca de las articulaciones costo-vertebrales y no a la mitad de las costillas, de esta manera, la pieza denominada "pecho" tuvo como base ósea la mayor parte de las cuatro costillas. Los cortes se agruparon según su valor comercial, como sigue. Cortes valiosos: punta trasera (porción proximal biceps femoris), muchacho redondo (semitendinosus), muchacho cuadrado (porción distal biceps femoris y parte del semimembranosus), pulpa negra (abductor, parte del recto internus, pectineus, semimembranosus), chocozuela (rectus femoris, parte del vastus lateralis, medialis e intermedius), pollo (tensor fasciae latae), lomito (psoas major y psoas minor, pequeñas porciones del quadratus lumborum e iliacus), solomo de cuerito (longissimus dorsi, longissimus costarum, intertransversales lumborum, trapezius y parte del serratus, rhomboideus y deltoideus), ganso (gluteus superficialis, medius y profundus). Cortes de mediano valor: lagarto de la reina (gastronemius), solomo abierto (latissimus dorsi, longissimus dorsi, multifidus dorsi, transversus espinalis, trapezius, parte del rhomboideus), paleta (deltoideus, infraspinatus, teres minor y major, coracobrachialis), papelón (supraspinotus). Cortes de bajo valor: falda (obliquus abdomis externi interni, rectus abdominis, cutaneus, transversus abdominis), lagarto con hueso anterior y posterior (digitorum longus, digitorum brevis, digitorum internus, digitorum externus, flexor carpi radialis, extensor carpi oblicuus), costilla con hueso (intercostales externi e interni, levatores costarum, retractor costae, transversus thoracis, rectus thoracis, longissimus costarum, porciones del longissimus dorsi, serratus dorsalis y scalenus), pecho con hueso (pectorales profundi y superficiales, porciones del brachiocephalicus y sternocefalicus). El promedio del producto del desposte de las medias canales, fue expresado de manera relativa (porcentaje del peso de la canal fría). Asimismo, se computaron la cantidad de hueso limpio, grasa recortada y recortes.
Los análisis estadísticos realizados fueron discriminados por condición sexual, haciendo uso de la versión 9.0 del Statistical Analysis System (SAS Institute, 2002). La tendencia central fue medida por la media aritmética; los estadísticos de dispersión considerados fueron el coeficiente de variación (CV), la desviación estándar (DE) y los valores mínimos y máximos de las variables.

El estudio incluyó un análisis de correlación con las variables medidas en la canal y el RCD. Para la correlación, se utilizó el coeficiente simple de Pearson (r). Para calificar los valores de r como altos, moderados o bajos, se utilizó el criterio convencional de Snedecor (alto, $\geq 0,7$; moderado, de 0,5 a 0,7 y bajo $\leq 0,5$ ). Para detectar la multicolinearidad y para evaluar las ecuaciones desarrolladas, se utilizó la opción VIF (factor de inflación de la varianza), DW (Durbin Watson) y ALL (todos) del procedimiento REG del SAS (2002). Se realizó además un análisis de residuos. Un análisis de regresión lineal múltiple se realizó con las variables que presentaron menos multicolinearidad (menor valor VIF), menos complejas de medir y las que más se asociaron con las variables dependientes, para así establecer una o varias fórmulas de predicción de las variables dependientes. El desarrollo de las ecuaciones predictivas se hizo a través de las opciones RSQUARE y STEPWISE del procedimiento REG del SAS (2002).

\section{Resultados y Discusión}

Los valores indican que la mayor variación se presentó en los índices grasos, siendo el espesor de grasa dorsal (ESPEGRASA) el rasgo con mayor variación (48,31\%); sin embargo, el acabado de grasa subcutánea (ACABADO) apreciado por puntuaciones, presentó un coeficiente de variación moderado $(27,43 \%)$, al igual que la conformación de la canal (CONFORMACION, $30,81 \%$ ) y el porcentaje de grasa renal (GRENAL, 24,52\%) (Cuadro 1). Mientras que la mayoría de las mediciones biométricas presentaron $\mathrm{CVs}$ menores que $10 \%$. En general, las submuestras mostraron la misma tendencia que se observa para la variación en las características de la muestra global (castrados más enteros). Sin embargo, para los búfalos enteros, los índices grasos corporales presentaron la mayor variación (hasta 51\%). También se observó una variación moderada de la CONFORMACION $(33,99 \%)$, pero, el resto de los rasgos de la canal presentó coeficientes de variación menores que $15 \%$. La escasa variación de las 
medidas corporales (longitudes y áreas) y la amplia variación de los indicadores de grasa corporal han sido reportadas por otros autores (Abraham et al., 1980; Huerta-Leidenz \& Morón, 1996; Huerta et al., 1997; Atencio 2001) para búfalos y bovinos.

En el Cuadro 2, se presentan los coeficientes de correlación simple para las características de las canales de búfalos (castrados y enteros) y el RCD, PHUESO y PGRASA. Para el peso de la canal (PCANAL), los valores de correlación (r) de RCD y PGRASA fueron bajos pero significativos $(\mathrm{p}<0,01)$, pero la correlación con PHUESO fue moderada. Los valores significativos de $r$ para PCANAL, en general, indican que animales más pesados rinden menos en cortes deshuesados con menor proporción de hueso y mayor proporción de grasa recortada. Con la excepción de GRENAL, los índices grasos tuvieron una asociación significativa de baja a moderada $(p<0,01)$ con RCD $(p>0,05)$. Los resultados muestran que aproximadamente $34 \%$ de la variación observada en el rendimiento en cortes de los búfalos es atribuida al ACABADO y $18 \%$ al ESPEGRASA. El ACABADO presentó asociaciones moderadas $(p<0,01)$ con RCD, PHUESO y PGRASA, indicando que a mejor ACABADO (menor valor numérico de acabado) habría menos RCD y PHUESO, pero el PGRASA sería mayor. Los resultados de este estudio sugieren que RCD, PHUESO y PGRASA son mejor estimados por la variable ACABADO, seguido de la medida de ESPEGRASA.

El área muscular, medida en la décima segunda costilla (AOL), no se asoció con RCD ( $p>0,05)$, pero se asoció de forma moderada a baja con PHUESO y PGRASA $(p<0,01)$, respectivamente. Con respecto a las medidas lineales en estudio, se observó que longitud de la canal (LCANAL), ancho del muslo (ANMUSLO) y circunferencia del muslo (CIRMUSLO) explican 23, 17 y $13 \%$ de la variación en RCD, respectivamente. Las variables longitud del muslo (LMUSLO) y la profundidad del tórax (PROTORAX) no se asociaron con RCD. En general, se encontró una asociación bajamoderada y significativa $(\mathrm{p}<0,01)$ de las medidas lineales de la canal con PHUESO y PGRASA. Los coeficientes de correlación de CONFORMACION fueron bajos $(\mathrm{p}<0,05)$ para $\mathrm{RCD}$, pero moderados para PHUESO y PGRASA. Los mismos indican que a mejor CONFORMACION se correlacionan menor RCD, PHUESO y más PGRASA.

A las características de la canal evaluadas no se le pueden atribuir, individualmente, más de $34 \%$ de la variación para RCD, 36\% para PHUESO y $46 \%$ para PGRASA. Con excepción de la CONFORMACION, se encontraron asociaciones negativas y significativas $(\mathrm{p}<0,05)$ del RCD con PCANAL, LCANAL, CIRMUSLO, ANMUSLO, LMUSLO, ESPEGRASA, GRENAL, PROTORAX y AOL; mientras que el ACABADO se relacionó positivamente $(\mathrm{p}<0,01)$ con el RCD. El PGRASA se asoció positiva y significativamente $(\mathrm{p}<0,05)$ con PCANAL, LCANAL, CIRMUSLO, LMUSLO, ESPEGRASA, GRENAL, PROTORAX, ANMUSLO y AOL; mientras que el ACABADO y la CONFORMACION tuvieron una asociación negativa y altamente significativa $(\mathrm{p}<0,01)$ con PGRASA. El PHUESO presentó correlaciones negativas $(\mathrm{p}<0,05)$ con todas las medidas biométricas.

De estos resultados se interpreta que a medida que las mediciones de la canal aumentaban, el RCD disminuía y PGRASA aumentaba. La asociación significativa $(\mathrm{p}<0,01)$ de ACABADO y LCANAL con RCD indica que a medida

Cuadro 1. Variación de diferentes características de canales de búfalos castrados y enteros ${ }^{(1)}$.

\begin{tabular}{|c|c|c|c|c|c|c|c|c|c|c|}
\hline \multirow[t]{2}{*}{ Variables } & \multicolumn{5}{|c|}{ Castrados } & \multicolumn{5}{|c|}{ Enteros } \\
\hline & Media & $\mathrm{DE}$ & $\mathrm{CV}$ & Mínimo & Máximo & Media & $\mathrm{DE}$ & $\mathrm{CV}$ & Mínimo & Máximo \\
\hline Peso canal caliente $(\mathrm{kg})$ & 210,45 & 37,97 & 18,04 & 135,00 & 271,00 & 222,75 & 41,00 & 18,40 & 166,00 & 293,00 \\
\hline Acabado exterior de grasa (puntos) ${ }^{(2)}$ & 2,79 & 0,83 & 29,83 & 1,00 & 4,00 & 2,95 & 0,75 & 25,37 & 1,00 & 4,00 \\
\hline Espesor de grasa dorsal $(\mathrm{cm})$ & 0,77 & 0,35 & 45,61 & 0,20 & 1,30 & 0,67 & 0,34 & 51,52 & 0,20 & 1,60 \\
\hline Grasa renal $(\%)$ & 1,69 & 0,34 & 20,29 & 0,96 & 2,36 & 1,71 & 0,48 & 28,45 & 0,95 & 2,96 \\
\hline Área muscular 12 da. costilla $\left(\mathrm{cm}^{2}\right)$ & 56,29 & 17,58 & 9,89 & 37,41 & 70,96 & 57,63 & 8,35 & 14,50 & 39,99 & 70,96 \\
\hline Longitud de la canal (cm) & 121,79 & 6,08 & 4,99 & 108,00 & 131,00 & 122,37 & 5,54 & 4,53 & 112,00 & 134,00 \\
\hline Profundidad de tórax $(\mathrm{cm})$ & 38,14 & 2,17 & 5,70 & 34,00 & 42,00 & 38,10 & 2,07 & 5,44 & 34,00 & 42,00 \\
\hline Circunferencia del muslo $(\mathrm{cm})$ & 110,08 & 7,68 & 6,98 & 97,00 & 122,00 & 111,79 & 9,04 & 8,09 & 97,00 & 127,00 \\
\hline Longitud del muslo $(\mathrm{cm})$ & 55,04 & 3,86 & 7,01 & 43,00 & 61,00 & 55,41 & 2,55 & 4,60 & 51,00 & 61,00 \\
\hline Conformación canal, puntos ${ }^{(3)}$ & 2,79 & 0,77 & 27,90 & 1,00 & 4,00 & 2,83 & 0,96 & 33,99 & 1,00 & 4,00 \\
\hline Rendimiento en cortes sin hueso $(\%)^{(4)}$ & 54,89 & 1,68 & 3,06 & 51,77 & 59,27 & 55,60 & 1,26 & 2,27 & 52,94 & 58,33 \\
\hline Porcentaje de grasa recortada $(\%)^{(5)}$ & 7,65 & 2,01 & 26,32 & 4,13 & 10,95 & 6,67 & 1,48 & 22,22 & 4,03 & 9,87 \\
\hline Porcentaje de hueso $(\%)^{(5)}$ & 12,11 & 2,19 & 18,11 & 10,43 & 16,07 & 12,41 & 1,01 & 8,17 & 10,67 & 14,31 \\
\hline
\end{tabular}

(1)Número de observaciones: 24; DE: desviación estándar; CV: coeficiente de variación (\%); (2)1: uniforme, 4: desprovisto. (3)1: excelente, 4: industria.

${ }^{(4)}$ Cortes al detal de alto, mediano y bajo valor. ${ }^{(5)}$ Con relación al peso de la canal caliente. 
que esas variables aumentaban, el RCD disminuía. El PHUESO mostró una relación $(\mathrm{p}<0,05)$ con PCANAL, ACABADO, AOL, PROTORAX, ANMUSLO, CIRMUSLO y CONFORMACION, lo que indica que a mayores valores de estas variables corresponde menor PHUESO. La relaciones observadas entre PGRASA y PCANAL, ACABADO, ESPEGRASA, LCANAL, ANMUSLO, CIRMUSLO y CONFORMACION muestran que a mayor valor numérico de estas variables, mayor es el PGRASA.

Trabajos realizados en vacunos (Crouse et al., 1975; Abraham et al., 1980; Hopkins \& Roberts, 1995; Atencio, 2001) señalan al PCANAL como una variable de poca fuerza predictiva para el RCD, resultando aún menor en búfalos (Huerta et al., 1997). En vacunos, algunos autores (Huerta-Leidenz \& Morón-Fuenmayor, 1996; Atencio, 2001) reportaron que la proporción de grasa interna de la canal explicaba más de $40 \%$ de la variación del RCD.

Los dos índices grasos medidos en este estudio (ACABADO y ESPEGRASA) no siguieron la tendencia esperada al asociarse positivamente con RCD, para cada condición sexual. En castrados, el ACABADO y ESPEGRASA tuvieron una asociación significativa ( $\mathrm{p}<0,01)$ con RCD; donde más de $20 \%$ de la variación observada en el RCD puede ser atribuida a estas características. En los búfalos enteros, solo el ACABADO llegó a influir en 35\% (p<0,01).

El AOL en búfalos castrados tuvo una asociación moderada $(\mathrm{p}<0,05)$ con RCD y PGRASA; mientras que en los enteros no se detectó esta asociación. Los resultados aquí reportados coinciden con: Atencio (2001), donde el AOL se asoció moderada y significativamente $(\mathrm{p}<0,01)$ con RCD y PGRASA, pero no se asoció con
PHUESO ( $\mathrm{p}>0,05)$ y con los resultados de Crouse et al. (1975), quienes indicaron que AOL, como indicativo de la muscularidad, es mejor índice predictivo del RCD que el PCANAL; y con Crouse y Dikeman (1976), con valores de AOL coincidentes al explicar el 16\% de la variación total del RCD.

La escasa variación de las medidas biométricas ha sido reportada en vacunos (Huerta-Leidenz \& MorónFuenmayor, 1996), y se conoce que en la medida en que un rasgo presente menos variación, pierde fuerza predictiva. En general, se encontró una asociación baja y significativa $(\mathrm{p}<0,01)$ de las medidas lineales de la canal con PHUESO y PGRASA, y algunas de ellas, como largo del muslo y de la media MUSLO no se asociaron ( $p>0,05$ ) con PHUESO. En el estudio de Huerta-Leidenz y Morón-Fuenmayor (1996), con canales venezolanas, también se evidenció la asociación baja $(\mathrm{p}<0,05)$ de las medidas lineales con el RCD, lo que está acorde con los resultados de Crouse \& Dikeman (1976), quienes indicaron que las medidas lineales de la canal como: LCANAL, longitud del tren posterior, LMUSLO, ANMUSLO, grosor y profundidad de la espalda tienen una relación positiva, de moderada a baja con el RCD.

Los coeficientes de correlación del perfil de CONFORMACION fueron bajos $(\mathrm{p}<0,01)$ para RCD, pero moderados para PHUESO y PGRASA. Los mismos coeficientes indican que cuanto mejor es la CONFORMACION, menor es el RCD, PHUESO y mayor el PGRASA. Este menor rendimiento, cuando se tiene un mejor perfil del muslo, corrobora los estudios previos, que aseguran que las estimaciones de conformación corporal por siluetas son influenciadas en sumo grado por la acumulación y distribución de grasa subcutánea que aumenta la corpulencia, disminuyendo

Cuadro 2. Coeficiente de correlación de Pearson (r) de las características de la canal de búfalos (castrados y enteros) y el rendimiento en cortes deshuesados (RCD), hueso y grasa.

\begin{tabular}{|c|c|c|c|c|c|c|c|c|c|}
\hline \multirow{2}{*}{ Variables independientes } & \multicolumn{3}{|c|}{ Castrados } & \multicolumn{3}{|c|}{ Enteros } & \multicolumn{3}{|c|}{ Total (enteros y castrados) } \\
\hline & $\mathrm{RCD}$ & Hueso & Grasa & $\mathrm{RCD}$ & Hueso & Grasa & $\mathrm{RCD}$ & Hueso & Grasa \\
\hline Peso canal caliente $(\mathrm{kg})$ & $-0,55^{* *}$ & $-0,52 * *$ & $0,58 * *$ & $-0,31^{\text {ns }}$ & $-0,65^{* *}$ & $0,54 * *$ & $-0,35 * *$ & $-0,57 * *$ & $0,49 * *$ \\
\hline Acabado de grasa (puntos) $)^{(1)}$ & $0,61 * *$ & $0,48 * *$ & $-0,67 * *$ & $0,59 * *$ & $0,66 * *$ & $-0,71 * *$ & $0,59 * *$ & $0,58 * *$ & $-0,68 * *$ \\
\hline Espesor de grasa dorsal $(\mathrm{cm})$ & $-0,44^{*}$ & $-0,63 * *$ & $0,57 * *$ & $-0,38^{\mathrm{ns}}$ & $-0,37^{\mathrm{ns}}$ & $0,42 *$ & $-0,43 * *$ & $-0,51 * *$ & $0,51 * *$ \\
\hline Grasa renal $(\%)$ & $-0,54 * *$ & $-0,63 * *$ & $0,69 * *$ & $-0,01^{\mathrm{ns}}$ & $0,05^{\mathrm{ns}}$ & $0,20^{\mathrm{ns}}$ & $-0,21^{\mathrm{ns}}$ & $-0,25^{\text {ns }}$ & $-0,39 * *$ \\
\hline Área muscular $12 \mathrm{da} .\left(\mathrm{cm}^{2}\right)$ & $-0,49^{*}$ & $-0,53 * *$ & $0,62 * *$ & $0,11^{\mathrm{ns}}$ & $-0,63 * *$ & $0,28^{\mathrm{ns}}$ & $-0,19^{\mathrm{ns}}$ & $-0,57 * *$ & $0,45 * *$ \\
\hline Longitud de la canal (cm) & $-0,57 * *$ & $-0,53 * *$ & $0,56^{* *}$ & $-0,48 *$ & $0,39^{\text {ns }}$ & $0,51 *$ & $-0,48 * *$ & $-0,47 * *$ & $0,50 * *$ \\
\hline Profundidad de tórax $(\mathrm{cm})$ & $-0,42 *$ & $-0,44 *$ & $0,43 *$ & $-0,04^{\mathrm{ns}}$ & $-0,59 * *$ & $0,32^{\mathrm{ns}}$ & $-0,24^{\mathrm{ns}}$ & $-0,50 * *$ & $0,37 * *$ \\
\hline Ancho del muslo (cm) & $-0,60 * *$ & $-0,54 * *$ & $0,67 * *$ & $-0,27^{\mathrm{ns}}$ & $-0,46^{*}$ & $0,49 *$ & $-0,41 * *$ & $-0,50 * *$ & $0,56 * *$ \\
\hline Circunferencia del muslo $(\mathrm{cm})$ & $-0,63 * *$ & $-0,58 * *$ & $0,75^{* *}$ & $-0,23^{\mathrm{ns}}$ & $-0,49^{*}$ & $0,48^{*}$ & $-0,36 * *$ & $-0,52 * *$ & $0,55^{* *}$ \\
\hline Longitud del muslo (cm) & $-0,43^{*}$ & $-0,42 *$ & $0,46^{*}$ & $-0,13^{\text {ns }}$ & $-0,26^{\text {ns }}$ & $0,26^{\mathrm{ns}}$ & $0,28^{\text {ns }}$ & $-0,37 * *$ & $0,36^{* *}$ \\
\hline Conformación canal (puntos) ${ }^{(1)}$ & $0,39^{\text {ns }}$ & $0,53 * *$ & $-0,53 * *$ & $0,32^{\mathrm{ns}}$ & $0,66^{* *}$ & $-0,51 * *$ & $0,33^{*}$ & $0,60 * *$ & $-0,52 * *$ \\
\hline
\end{tabular}

${ }^{(1)}$ Coeficiente de correlación simple de Spearman $(\mathrm{rs}) .{ }^{\mathrm{ns}}$ No significativa. $*$ y $* *$ Significativa a 5 y $1 \%$ de probabilidad, respectivamente. 
la proporción de cortes valiosos de la canal, una vez recortada la grasa en exceso.

Estos resultados también sustentan los de Apple et al. (1991), Crouse \& Dikeman (1976), quienes trabajaron con novillos, y coinciden en que la muscularidad o conformación del tren posterior, aun estando correlacionada significativamente, tuvo un coeficiente de poca magnitud con el RCD.

En el estudio de Huerta-Leidenz \& Morón-Fuenmayor (1996), la conformación no se relacionó con RCD, y ello puede ser explicado por la influencia que pudo haber tenido la incorporación de las vacas en el estudio señalado, dada la tendencia en las vacas de mostrar alta asociación de RCD y conformación. El argumento anterior se apoya en Hodgson et al. (1992), quienes detectaron que la muscularidad general tenía un efecto significativo en el porcentaje $\operatorname{RCD}\left(r^{2}=-0,80\right)$ en canales de vacas.

Estos resultados difieren, con relación al rendimiento en cortes, de los presentados por Huerta-Leidenz et al. (1997), quienes mostraron que en búfalos enteros, el rendimiento en cortes de valor sólo se asociaba con el ancho del muslo. Conviene aclarar que en ese estudio el rendimiento predicho involucraba algunos cortes deshuesados de bajo valor.

El PCANAL difirió su grado de asociación dependiendo de la condición sexual; que en búfalos castrados y se observó hubo una asociación moderada con el RCD y PGRASA ( $\mathrm{p}<0,05)$, y en búfalos enteros, una asociación baja con PGRASA. Atencio (2001) encontró que PCANAL tiene baja fuerza predictiva en el RCD y PHUESO, y nula para el PGRASA, y destacó que los animales más pesados rinden más cortes de valor y menos en la proporción de hueso. Otros autores (Crouse et al., 1975; Abraham et al., 1980) señalan al PCANAL como una variable de poca fuerza predictiva para el RCD, y algunos estudios han sido criticados por utilizar el PCANAL como variable independiente de las ecuaciones de predicción del rendimiento.

Los dos índices grasos medidos en este estudio (ACABADO y ESPEGRASA) no siguieron la tendencia esperada al asociarse con RCD en cada condición sexual. En vacunos, Atencio (2001) encontró que más de $20 \%$ de la variación, observada en el rendimiento en cortes para la venta de bovinos venezolanos, puede ser atribuida a su regresión lineal simple sobre ACABADO, el ESPEGRASA o el GRENAL, y sus valores $r$ indican que a medida que aumenta la grasa, se reduce el RCD y el PHUESO, aunque la GRENAL fue el mejor estimador, seguido del ESPEGRASA. Huerta-Leidenz \& Morón-Fuenmayor (1996) reportan que la proporción de grasa interna de la canal explicaba $42 \%$ de la variación del rendimiento en cortes de valor. En los Estados Unidos de Norteamérica, se ha reportado que el espesor de grasa dorsal resulta ser el mejor estimador del RCD (Crouse et al., 1975; Crouse \& Dikeman, 1976; Abraham et al., 1980; Apple et al., 1991). Sin embargo, en esos estudios, el valor original del ESPEGRASA fue ajustado por el acabado exterior, algo no contemplado en el presente estudio.

Por multicolinearidad, se descartaron algunas variables involucradas en las ecuaciones, reduciendo el número final de rasgos de la canal, inicialmente considerados por su asociación con las variables dependientes. El Cuadro 3 muestra los valores $\mathrm{R}^{2}$ y los coeficientes de Mallows ( $\mathrm{Cp}$ ) de las posibles regresiones que mejor predicen el RCD, PHUESO y PGRASA para el total de búfalos, para los castrados y enteros. El modelo que tenía mayor ajuste $(\mathrm{Cp}=3,03)$ y explicaba $39 \%$ de la variación en RCD involucró 5 variables: ACABADO, AOL, ANMUSLO, LCANAL y GRENAL. Las variables CONFORMACION, ESPEGRASA y GRENAL explicaron 40\% (Cp = -1,54) de la variación en PHUESO; mientras que para la predicción de PGRASA, las variables ACABADO, ESPEGRASA, ANMUSLO y GRENAL explicaron 70\% $(\mathrm{Cp}=4,01)$. $\mathrm{Al}$ introducir la condición sexual como variable en los modelos de regresión, para la predicción de RCD, PHUESO y PGRASA para el total de búfalos, los valores $\mathrm{R}^{2}$ no aumentaron, y los coeficientes $\mathrm{Cp}$ no ajustaron mejor al número de variables dentro de cada modelo de regresión. Reiling et al. (1992) trabajaron con una muestra que incluyó toros, novillos y novillas, y observaron incrementos en los valores $\mathrm{R}^{2}$ en sus ecuaciones, al incluir la condición sexual en el modelo de regresión.

Los estudios de Murphey et al. (1985) demostraron que las ecuaciones de predicción para rendimiento en cortes, derivados de la mezcla de datos provenientes de novillas y novillos, disminuían la fuerza predictiva de la 
ecuación. Entre tanto, enfatizan con sus resultados que el uso de una mayor variedad de tipos raciales y grupos sexuales mejoraban la estimación de la ecuación para ganado vacuno de carne. Más recientemente, Lee et al. (2005) mostró que era necesario desarrollar una ecuación de predicción propia para el tipo de ganado nativo Koreano (Hanwoo), ya que el uso de otras ecuaciones foráneas producía predicciones equívocas y subestimadas. Para el caso de los búfalos, es razonable pensar que la ecuación de predicción proveniente de ganado vacuno no puede ser de utilidad y, por ello, es necesario el desarrollo de ecuaciones específicas para esta especie. En este caso, la inclusión de la clase sexual no mejoró los valores de $\mathrm{R}^{2}$ y $\mathrm{Cp}$ de la ecuación, por lo que se presentan las ecuaciones para cada condición sexual.

Para búfalos castrados, al menos 54\% (Cp=3,35) de la variación observada en RCD se atribuyó a su regresión lineal sobre ACABADO, CONFORMACION, ESPEGRASA, ANMUSLO, y CIRMUSLO. La CONFORMACION, ESPEGRASA, LMUSLO, CIRMUSLO y GRENAL explicaron solo 63\% $(\mathrm{Cp}=4,46)$ de la variación en PHUESO. El ACABADO, ESPEGRASA, ANMUSLO, CIRMUSLO y GRENAL se atribuyeron al menos $85 \%(\mathrm{Cp}=5,13)$ de la variación en PGRASA.

Para los búfalos enteros, al menos $65 \%(\mathrm{Cp}=2,22)$ de la variación observada en RCD se atribuyen a su regresión lineal sobre ACABADO, AOL, ANMUSLO, LCANAL y PROTORAX. El ACABADO, AOL, LMUSLO, PROTORAX y PCANAL explican $78 \%$ $(\mathrm{Cp}=1,70)$ de la variación en PHUESO. Para el ACABADO, LMUSLO, ANMUSLO, LCANAL y GRENAL se atribuyen $76 \%(\mathrm{Cp}=2,98)$ de la variación en PGRASA.

El análisis de multicolinearidad realizado demostró que en ninguna de las ecuaciones señaladas en los cuadros anteriores se evidenció influencia de multicolinearidad entre variables. Los valores de inflación de la varianza y los valores de los coeficientes de Durbin Watson fueron todos cercanos a la unidad, que en general indican la validez estadística de los resultados planteados. El análisis de residuos no indicó la existencia de patrones de comportamiento que impliquen alguna violación de la suposición de homocedasticidad, al arrojar valores significativos $(\mathrm{p}<0,05)$ de los coeficientes de Cook. Puede decirse, entonces, que los modelos de regresión lineal 
múltiple desarrollados pueden ser utilizados en previa validación, para predecir las variables dependientes estudiadas.

La mejor ecuación para castrados fue: $\mathrm{RCD}_{\text {castrados }}=83,764+0,824($ ACABADO $)-$ 1,336(CONFORMACION) $+1,019$ (ESPEGRASA) 0,160(ANMUSLO) - 0,078(CIRMUSLO), con $\mathrm{R}^{2}=0,541$ y Cp-Mallows $=3,35$; y para enteros $R C D_{\text {enteros }}=55,530$ $+1,062(\mathrm{ACABADO})+0,079(\mathrm{AOL})-0,109$ (ANMUSLO) $-0,067\left(\right.$ LCANAL) $+0,189$ (PROTORAX), con $^{2}=0,658$ y Cp-Mallows $=2,21$.

\section{Conclusiones}

1. Las ecuaciones desarrolladas para búfalos enteros y castrados logran explicar más de 50\% de la variación del RCD; aunque las ecuaciones para PGRASA y PHUESO tuvieron mayor fuerza predictiva.

2. Los resultados de este estudio enfatizan la necesidad de se obtener mejores estimadores del rendimiento, con uso de una mayor variedad de condiciones sexuales y pesos de mercado, para lograr una mayor aplicabilidad industrial.

\section{Agradecimientos}

Al Consejo de Desarrollo Científico y Humanístico (Condes) de la Universidad del Zulia y a la Asociación de Búfalos de Venezuela (Asobufalo), por el financiamiento del proyecto; al personal de investigación del Grupo de Investigación en Carnes de la Universidad del Zulia (Carnes-LUZ), por la colaboración en la ejecución del ensayo.

\section{Referencias}

ABRAHAM, H.C.; MURPHEY, C.E.; CROSS, H.R.; SMITH, G.C.; FRANKS JÚNIOR, W.J. Factors affecting beef carcass cutability: an evaluation of the USDA yield grades for beef. Journal of Animal Science, v.50, p.841-851, 1980.

AMADOR-GÓMEZ, I.; PALACIOS-GÓMEZ, A. Informe final proyecto sistemas de clasificación de canales bovinas, carne y comercialización en Colombia. Santafé de Bogotá: Universidad Nacional da Colombia, 1994.

APPLE, J.K.; DIKEMAN, M.E.; CUNDIFF, L.V.; WISE, J.W. Determining beef carcass retail product and fat yields within 1 hour postmortem. Journal of Animal Science, v.69, p.4845-4857, 1991.

ATENCIO, O. Predicción del rendimiento en cortes de carnicería de bovinos venezolanos. 2001. 105p. Tesis (M.Sc.) - Universidad del Zulia, Maracaibo.
COMISIÓN VENEZOLANA DE NORMAS INDUSTRIALES. Norma 0792-82: carne de bovino: definición e identificación de las piezas de una canal. Caracas, 1982. 11p.

CROUSE, J.D.; DIKEMAN, M.E. Determinates of retail product of carcass beef. Journal of Animal Science, v.42, p.584-591, 1976.

CROUSE, J.D.; DIKEMAN, M.E.; KOCH, R.M.; MURPHY, C.E. Evaluation of traits in the U.S.D.A. yield grade equation for predicting beef carcass cutability in breed groups differing in growth and fattening characteristics. Journal of Animal Science, v.41, p.548553, 1975.

JARDIM, P.O.C.; DODE, M.A.N.; OSORIO, J.C.S.; LÜDER, W.E. Estimativa da composição física em carcaças de novilhos holandês PB. Pesquisa Agropecuária Brasileira, v.26, p.1193-1199, 1991.

HODGSON, R.R.; BELK K.E.; SAVELL J.W.; CROSS H.R.; WILLIAMS, F.L. Development of a multivariate yield grade equation to predict compositional traits in mature cows carcasses. Journal of Animal Science, v.70, p.2159-2166, 1992.

HOPKINS, D.L.; ROBERTS, A.H.K. The value of carcass weight, fat depth measures and eye muscle area for predicting the percentage yield of saleable meat in Australian grass-fed beef carcasses for Japan. Meat Science, v.41, p.137-145, 1995.

HUERTA-LEIDENZ, N. La experiencia venezolana en la implantación de sistemas de clasificación de ganado y canales bovinas. En: GONZÁLEZ-STAGNARO, C.; SOTO-BELLOSO, E.; RAMÍREZ-IGLESIA, L. (Ed.). Avances en la Ganadería de Doble Propósito. Maracaibo: Astro Data, 2002. p.601-621.

HUERTA-LEIDENZ N.; MANSUTTI, D.; RODAS-GONZÁLEZ, A. Variación de características de la canal de búfalos y vacunos enteros, cebados a pastoreo y correlaciones con el rendimiento en cortes valiosos, grasa recortada y hueso. Archivos Latinoamericanos de Producción Animal, v.5, p.577-579, 1997. (Suplemento 1)

HUERTA-LEIDENZ, N.; MORÓN-FUENMAYOR, O. Variación de características en pie y en canal de bovinos en Venezuela y su relación con el rendimiento de cortes valiosos. Revista Científica, v.6, p.53-57, 1996.

LEE, J.M.; YOO, Y.M.; PARK, B.Y.; CHAE, H.S.; HWANG, I.H.; CHOI, Y.I. A research note on predicting the carcass yield of Korean native cattle (Hanwoo). Meat Science, v.69, p.583-587, 2005.

MALAVER, Y.; CALZADILLA, S.; RODAS-GONZÁLEZ, A.; GONZÁLEZ, J.; MANSUTTI, D.; MORENO, D.A.; HUERTALEIDENZ, N. Estudio preliminar para validar el sistema venezolano de clasificación de canales bovinas con toros sacrificados en la región oriental: estados Monagas y Anzoátegui. Revista Científica, v.10, p.468-479, 2000.

MULDOWNEY, D.; CONNOLLY, J.; KEANE, M.G. Compositional data analysis in the study of carcass composition of beef cattle. Livestock Production Science, v.67, p.241-250, 2001.

MURPHEY, C.E.; JOHNSON, D.D.; SMITH, G.C.; ABRAHAM, H.C.; CROSS, H.R. Effects of sex-related differences in external fat deposition on subjective carcass fatness evaluations-steer versus heifer. Journal of Animal Science, v.60, p.666-674, 1985.

REILING, B.A.; ROUSE, G.H.; DUELLO, D.A. Predicting percentage of retail yield from carcass measurements, the yield grading equation, and closely trimmed, boxed beef weights. Journal of Animal Science, v.70, p.2151-2158, 1992. 
RODAS-GONZÁLEZ, A. Limitantes y vicios del sistema de categorización venezolana de canales bovinas. En: GONZÁLEZSTAGNARO, C.; SOTO-BELLOSO, E. (Ed.). Manual de Ganadería Doble Propósito. Maracaibo: Astro Data, 2005. p.654-659.

SAS INSTITUTE. SAS/STAT: user's guide statistics. Cary: SAS Institute, 2002.

SCHINDLER, V.; PRUZZO, L.; OLIVERA, M.L.; GRIGERA NAÓNA, J.J.; ABBIATTI, N.; DE SANTA COLOMA, L.F.
Predicción de rendimiento de cortes minoristas de reses bovinas en Argentina. Archivos Latinoamericanos de Producción Animal, v.12, p.105-111, 2004.

SHACKELFORD, S.D.; CUNDIFF, L.V.; GREGORY, K.E.; KOOHMARAIE, M. Predicting beef carcass cutability. Journal of Animal Science, v.73, p.406-413, 1995.

VENEZUELA. Decreto presidencial n.1896. Gaceta Oficial de la República de Venezuela, Caracas, Venezuela. 4p. 1997.

Recibido el 15 de mayo de 2007 y aceptado el 9 de noviembre de 2007 